\title{
IMAGE SEGMENTATION BY SOBEL EDGE DETECTION ALGORITHM - MOSAIC METHOD
}

\author{
Ratko Ivkovicín, \\ Ivana Milošević2, \\ Mile Petrović, \\ Petar Spalević, \\ Stefan Panić
}

\author{
${ }^{1}$ Faculty of Technical Sciences, \\ University of Pristina, \\ Kosovska Mitrovica, Serbia \\ ${ }^{2}$ The School of Electrical and Computer \\ Engineering of Applied Studies, \\ Belgrade, Serbia \\ ${ }^{3}$ Singidunum University, \\ Belgrade, Serbia \\ ${ }^{4}$ Faculty of Natural Sciences and Mathematics, \\ Kosovska Mitrovica, Serbia
}

\begin{abstract}
:
Segmentation is one of the latest directions of digital imaging development, presented by partial segments which are parts of the same image. The currently used algorithms are rare and far from ideal. Depending on the situation, algorithms behave differently, so it is almost impossible to single out a unique conclusion. This paper gives a proposal for new segmentation techniques for the digital image processing with auxiliary Sobel edge detection algorithm and specially a defined threshold of detection. Matlab software is used for giving a particular conceptual design of a partial display of images and for correlating adequate numerical methods of digital processing. The study results confirm the quality of the proposed algorithm and position it for practical use and they also give guidelines for further improvements.
\end{abstract}

\section{Keywords:}

digital image processing, sobel edge detection, image segmentation, mosaic method.

\section{INTRODUCTION}

The problems of digital image segmentation and grouping segments represent a major challenge for the entire field of Computer Vision and Digital Image Processing $[1,2]$. These problems do not exist only in the area of Multimedia [3]. In 1938 Werheimer defined the visual perception of what humans saw as a group of small segments of the visual field [4]. Medically speaking, while the left hemisphere of the brain tries to represent a picture of segments, the right hemisphere represents it as a whole. A digital image can be defined in a very similar way as a set of segments in a defined resolution $\mathrm{m}^{\star} \mathrm{n}$ that does not overlap. Here lies an important emphasis, there must not be any overlaps between image segments [5], or the process of image formation will go in the opposite direction. The complete human visible spectrum (380-780 nm), which is viewed through the lens, is converted through the sensor into three different signals ( $R, G, B)$. And for this reason, a major problem is how to separate visually identifiable segments by using arithmetic and logical tools. Actually, image segments can be extracted as regions between defined discontinuities in the image. The segmentation techniques based on discontinuity fundamentally rely on a sudden change in the value of intensity between pixels [6]. These methods are called "Edge or Boundary" 
based methods [7]. Edge is a set of linked pixels at the border between different regions in which some image parameters are 'breaking', losing, their values. These parameters can be defined as the difference in the shade of gray, different textures, etc.

In many algorithms Edge detection is used for each channel separately, but results must be presented as a sum or as a gradient of all observed channels. According to all of the above, edge detection algorithms may be classified into two categories:

1. Parallel detection method implies that the set of points (pixels) on the border are independent whether or not other points lie on that edge. This method is carried out separately for all three channels. Spatial frequency filtering is most important during this procedure. There are various types of parallel differential operators such as Roberts, Sobel and Prewit. They are defined as the real differential operators and Laplacian algorithms. The main differences between these operators are masks which are based on the performed detection.

2. Sequential edge detection uses points (pixels) as the central part of the algorithm. Properly chosen, a pixel can influence the outcome of this process. There are a number of consecutive techniques that use sequential search techniques with a dynamic program.

Besides this theoretical framework, which is needed to understand the new algorithm, this work has given a methodological framework for its realisation as well as the type of work in different real situations. A proposal for new digital image segmentation techniques done by using Sobel edge detection algorithm, RGB colour model, alpha composite process, full gray scale and a combination of parallel and sequential edge detections is given in this article. One of the main innovations in the proposed image segmentation technique is the central role of the edge detection algorithm. The complete proposed solution is fully compatible with other edge detection algorithms, so it is adjustable for particular situations.

\section{THEORETICAL FRAMEWORKS}

When the Mosaic method of image segmentation is used, the selection of the algorithm for edge detection is a very important part. The segmentation is done on areas located between defined edges. Numerous research papers define the Sobel algorithm as one of the best algorithms for edge detection in medicine, signal transmission in optical cables, forensic, photogrammetry, etc. $[8,9,10]$. The function of the low-level detection is the most commonly used function of the Sobel edge operator. Also, because of its simplicity, this Sobel algorithm is the most frequently used model for a large number of other algorithms. This function represents a discrete differential operator based on the independent $2 \mathrm{D}$ gradients and masks, by which the edges are detected. Independent gradients are calculated separately for the $\mathrm{x}$ and $\mathrm{y}$ axes:

$$
\begin{aligned}
& \text { Img=imread('Lena.tiff'); } \\
& {[m, n, k]=\text { size(Img);. }}
\end{aligned}
$$

In mathematical terms, the image is divided into gradients (for $\mathrm{x}$ and $\mathrm{y}$ axes) which are calculated on the basis of the $3 \times 3$ mask:

$$
\begin{aligned}
& \text { for } i=1 \text { : size }(m)-2 \\
& \text { for } j=1 \text { : size }(n)-2 \\
& G x=\left|\begin{array}{ccc}
1 & 0 & -1 \\
2 & 1 & -2 \\
1 & 0 & -1
\end{array}\right| * \text { image } \\
& G y=\left|\begin{array}{ccc}
1 & 2 & 1 \\
0 & 0 & 0 \\
-1 & -2 & -1
\end{array}\right| * \text { image }
\end{aligned}
$$

end

end

In this way the overall gradient is calculated as a value $G(m, n)=\sqrt{(G x)^{2}+(G y)^{2}}$.

One of the reasons for the popularity of the Sobel operator is its capability of processing $\mathrm{n}$-dimensional spaces. This is especially important for analysis in $3 \mathrm{D}$ spaces (three-dimensional technology and image processing) $[11,12,13]$. After selecting the algorithm for edge detection, it is necessary to determine the format for the extraction of the image segments. In comparison to other formats, Portable Network Graphics (PNG) format is selected for the purpose of this work due to its advantages.

Fig. 1 represents the influence of lossless data compression in PNG format (left image) compared to JPEG image (right image) format at the same compression ratio. PNG image format is based on 24 bits (32 or 48 bit) with full gray scale and alpha composite process, as the most important characteristics for this work. 
Transparent part of the image presented as gray and white cubes on Figure 2 (left image). Alpha composite process and quality of edge detection are responsible for choosing PNG as the most acceptable image format. An alfa composite process combines the image with the background to create a partial or complete transparency of the image. This attribute is very important and takes its full meaning in VRML at MPEG4 video format.

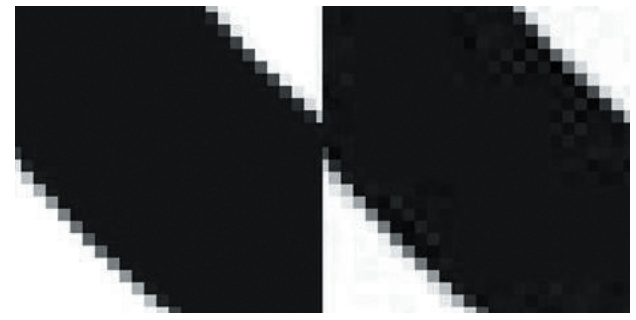

Fig. 1. Comparative review of image at the same compression level, PNG (left) and JPEG (right)

A PNG image format allows separation of individual objects and different processes on each segment. Defining the discontinuity of edges can be the first problem in the process of image segmentation. According to this, it is very important to find the right algorithm with optimal sensitivity.

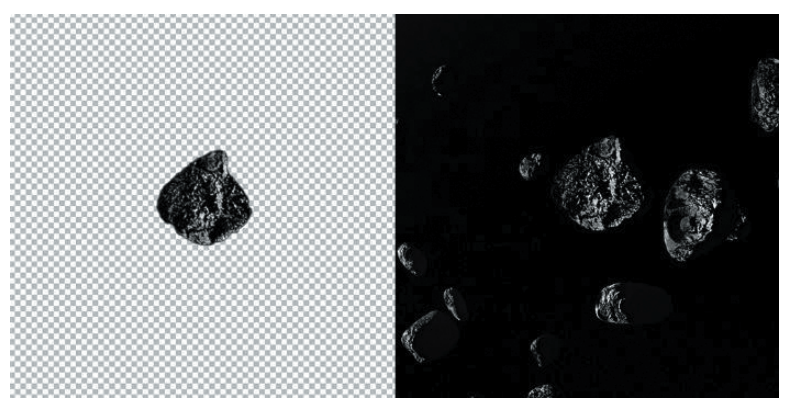

Fig. 2. Difference between PNG (left) and JPEG (right)
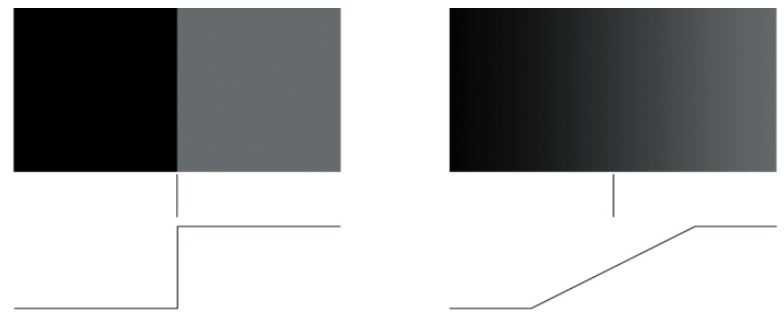

Fig. 3. Model of ideal edge detection (left); real situation in the detection of edges (right)
Practically speaking, in real situations cases of ideal edge detections (Figure 3 ) are rare. In the majority of cases, the edge detection depends on the part of the curve where a threshold is set. In this way the main goal of the algorithm for edge detection is to define the sensitivity of the threshold. For example, in the case of a high level of details, it is advisable to use algorithms with a lower level of detection [14]. Defining the threshold according to the user, depending on the situation, represents an ideal solution (through a mask detection or in any other way) [15].

\section{METHODOLOGY}

According to the previous paragraphs, an image segment can be defined as an area between image edges. These areas in an image, after an edge detection operator is applied, are marked as black. Because of that, in order to detect segments, it is necessary to compare the resulting image with the original image. This process contains two different images with the same resolution. An image with clearly defined polygons (Figure 4a), an image with medium level of detail (Figure $4 \mathrm{~b}$ ) and an image with high level of detail (Figure 4c) are given as referent images.
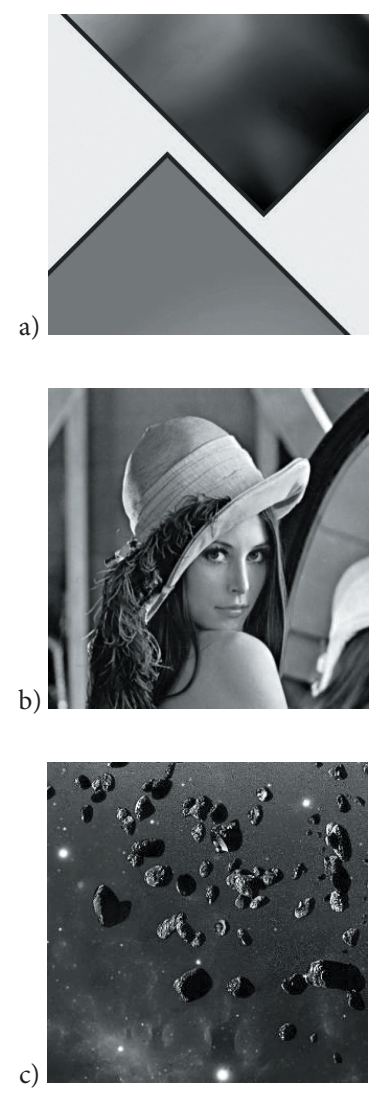

Fig. 4. Original images: a) Polygons, b) Lena, c) Space 
Due to the synchronization with similar research papers, all original images are in TIFF format, with bit depth from $300 \mathrm{dpi}$ and resolution $512 \times 512$. The image with clearly detected edges is the base for the segments, but these segments belong to the original image. The entire process of segregation of image segments is shown schematically in Figure 5.

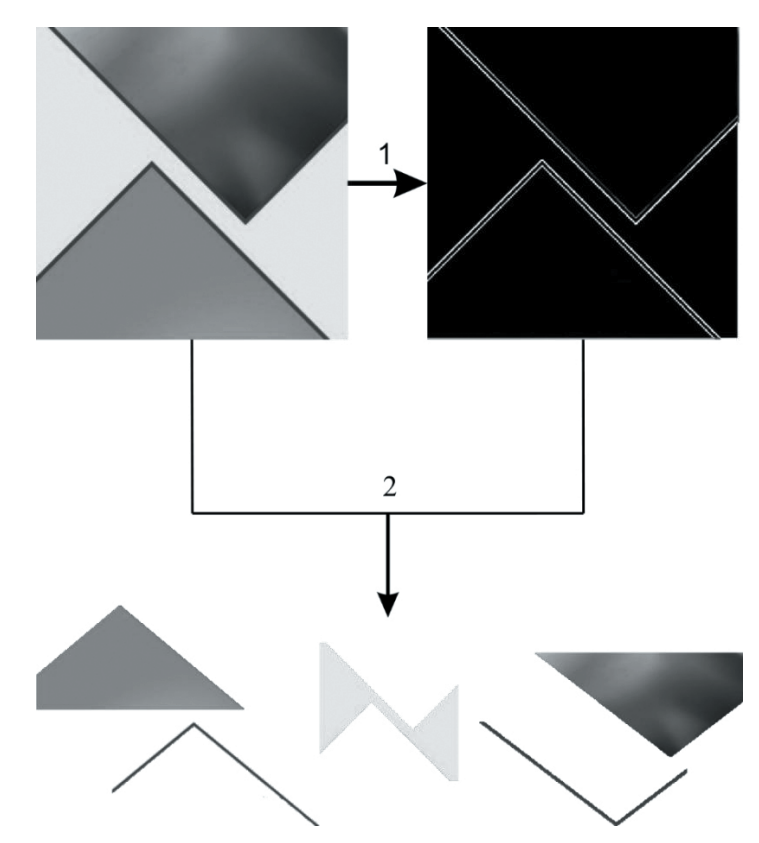

Fig. 5. Segmentation block diagram based on the edge detection algorithm

The processes in segregation of image segments are labeled with numbers 1 and 2 (Figure 5). In the first process, the original image with the help of the Sobel algorithm, is being converted into an image with clearly defined edges. Now, the original image and the image with clearly defined edges are being used for process 2 . The Segregation of the image segments is performed with the help of the Matlab software package, especially the Image Processing Toolbox and defined threshold (125). The value of 125 is taken as a half of the maximum pixel values from completely black to white in 8-bit image format. The Sobel edge detection algorithm is based on the gradient. In this way, the surroundings of the edges are strictly defined but not and the edges. According to this, the threshold of 125 is defined to serve as an eliminator of the side edge parts. In case the segmentation is carried out with another edge algorithm, it is possible to reduce or increase a threshold value [16]. For example, the Canny algorithm (which is based on the Sobel algorithm) is much more particular in terms of edges and in that case, it is possible to decrease a threshold value [17]. Every image has $m^{\star} n$ resolution (number of pixels), where $m$ represents the vertical line and $n$ the horizontal line of the image. The algorithm compares the pixels of the original image with the image of clearly defined edges, element by element in an $\mathrm{m}^{\star} \mathrm{n}$ matrix. In the first cycle, $m=1$, and if the value of the current pixel is less than 125 :

$$
\begin{aligned}
& \text { if }(m, n) \operatorname{Img}<125 \\
& \operatorname{Img} s g(X)=\operatorname{Img} \text { _Org }(m, n) \text {; } \\
& \text { end }
\end{aligned}
$$

then the newly created image Img_sg $(X)$ takes the value of the pixel from the original image. The variable $\mathrm{X}$ represents the number of segments, and the value $\max (X)$ represents the total number of segregated segments. If the value of the observed pixel is higher than 125 , the value of the horizontal (n) increases by one. This procedure is repeated until values for $m$ and $n$ parameters become higher than 125 . In that case, the variable $\mathrm{X}$ increases by one and creates a new segment.

It is very important to describe the resolution of the recorded segments, and the extracting image format. From the reasons mentioned above, we can conclude that PNG format is the best solution, especially because of:

- The track of the edges - the entire concept of segmentation is based on an algorithm for the edge detection,

- The alpha composite process - transparent parts of image background.

These two facts are very important for the segregation of image segments. The highest pixel position from the vertical is taken as a vertical reference and the same is done for the horizontal.

Isolated segments do not have a square (or rectangular) resolution but they must be placed in the image with a square or rectangular resolution, and the missing pixels must be written during the alpha composite process. The alpha composite record will write transparent parts of the image as the supplement of the square (rectangular) resolution. For example, a segment $\mathrm{X}$ that has a resolution of $\mathrm{mx}$ and $\mathrm{nx}$ will be defined through a separate segment and a transparent area as a supplement to the square resolution. At the end of the algorithm, one should take into account the mode of extracting, especially when defining the value of alpha composite track 
segments in PNG format. An alpha composite record should have a value of 1 (maximum value) which represents completely transparent background characteristics, and is defined as: imwrite (X, 'segment.png', 'Alpha', $A) ;$. In the previous expression, parameter A defines an alpha composite part of a digital image.

\section{RESULTS \& DISCUSSION}

The image selected for the analysis is the image with a low-level of detail (image with precisely arranged polygons) shown in Figure 6a, the image with a medium level of detail Figure $6 \mathrm{~b}$ and the image with a high level of detail in Figure $6 c$. The main idea for this kind of image selection is to determine algorithm behavior which depends on the situation of a low level of detail (clearly defined polygons), a medium and a high-level of detail.

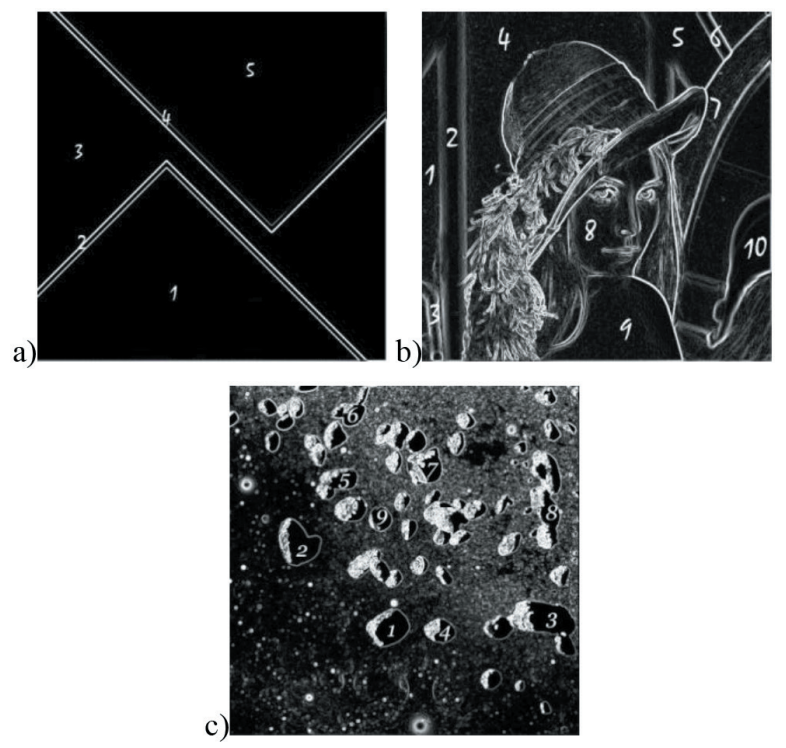

Fig. 6. The areas between the edges marked with numbers: a) image with a small level of detail, b) image with a medium level of detail, c) image with a high level of detail

According to Table 1, the Level of Detail (LoD) of the image in Figure $4 \mathrm{~b}$ (Lena) is by $64 \%$ higher than that of the image in Figure 4a (polygons). It is logical to expect that images with a lower level of detail give more precisely defined edges than the images with high LoD [18].

\begin{tabular}{cccc}
\hline LoD & Polygons & Lena & Space \\
\hline DCT & 1.5065 & 2.3296 & 3.0752 \\
\hline
\end{tabular}

Table 1. The level of detail for Figure $3 \mathrm{a}$, Figure $3 \mathrm{~b}$ and Figure $3 \mathrm{c}$ according to DCT

(Discrete Cosine Transformation)

Furthermore, another reflection of the quality of the selected image is the high degree of Entropy as a measure of the image potential $[19,20]$. Both images show the value of Entropy which is over the half of the maximum value for 8 -bit image format.

This analysis has shown that the better results can be expected for images with clearly defined polygons than for the images with high LoD. This can be clearly demonstrated by the fact that discontinuity between the picture parameter suddenly occurs in the image with clearly defined polygons. In the image with a high LoD the transition between the image discontinuities comes more slowly.

\begin{tabular}{cccc}
\hline Entropy & Polygons & Lena & Space \\
\hline bit & 5.4308 & 7.7502 & 7.9756 \\
\hline
\end{tabular}

Table 2. The level of Entropy for Figure 3a, Figure $3 \mathrm{~b}$ and Figure $3 \mathrm{c}$ according to DCT

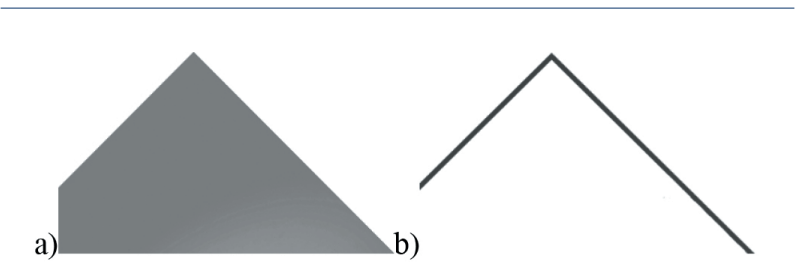

d)

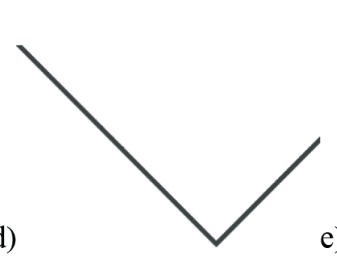

e)

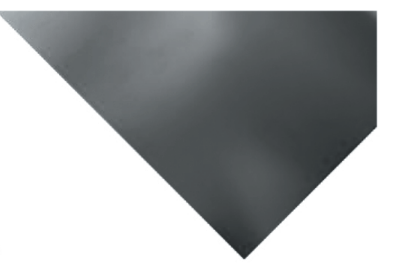

Fig. 7. Segmentation of image "Polygons" 

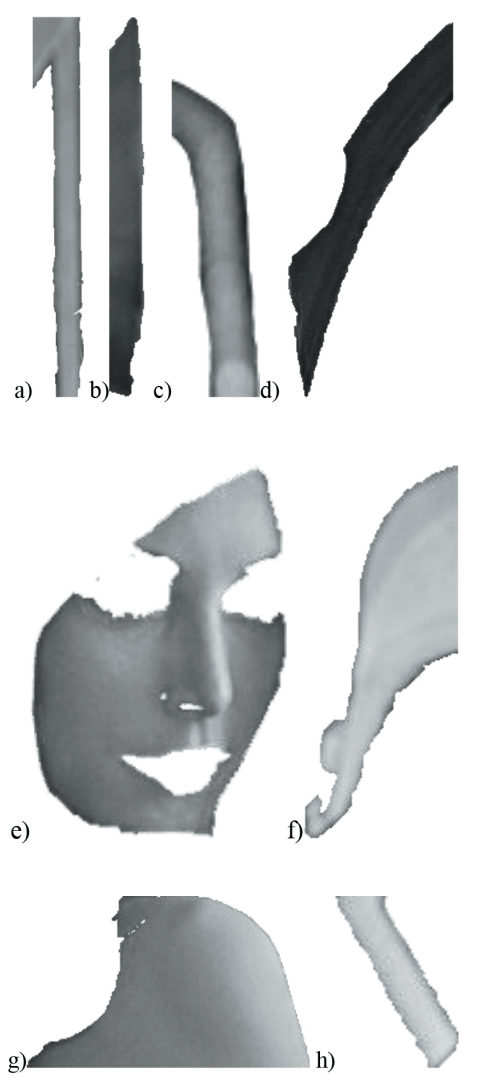

Fig. 8. Segmentation of image "Lena"

Figure 7, Figure 8 and Figure 9 show the separate segments from Figure $3 \mathrm{a}$, Figure $3 \mathrm{~b}$ and Figure $3 \mathrm{c}$ respectively. For the reasons which have already been explained, the segments which are located in Figure 3 are of the higher quality and they are visually better. Figure 3 a consists of only 5 segments, while Figure 3 b consists of more than 40 different segments, Figure $3 \mathrm{c}$ contains more than 80 . For the purpose of this analysis only 8 large segments of Figure $3 \mathrm{~b}$ and Figure $3 \mathrm{c}$ are presented. In most cases it is very important that separated segments are visually recognizable.

Besides the obvious visual results, it is difficult to measure mathematically the quality of the obtained segments. The lack of adequate parameters for measuring is the main problem in these situations. The first problem comes from the resolution of extracted segments. Measuring the results obtained through the parameters is not relevant to:

- PSNR - it estimates the quality of the transmission segment in relative to the original image,

- SNR - it is not relevant to measure the signal noise ratio of the complete image in relation to its segment, because the sum is not equally distributed in the image,
- MSE - because of the same reason as PSNR,

- SSIM, MSSIM, DSSIM - the structure of the obtained segments do not correspond to the structure of the original image,

- LoD - defined as numbers of changes on the observed image parameter. These changes are not of the constant value in the image, and because of that it is irrelevant to compare LoD of one segment to the LoD of the whole image.

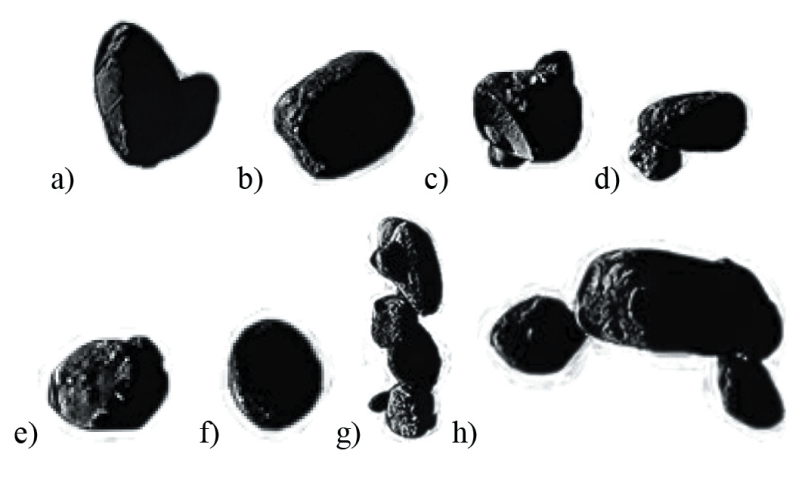

Fig. 9. Segmentation of image "Space"

If transparent parts of the image are excluded, Entropy can be partially taken into analysis for obtained segments. Entropy is the only image parameter that has a constant value throughout the observed image. Thus, every part of the image must have the same value of Entropy. If transparent parts of the image are taken into consideration, the value of Entropy of each segment will be less than the Entropy of the original image. Table 3 confirms previous claim.

\begin{tabular}{cccc}
\hline $\begin{array}{c}\text { Entropy } \\
\text { (bit) }\end{array}$ & Polygons & Lena & Space \\
\hline Segment 1 & 2.7447 & 4.5862 & 3.0087 \\
\hline Segment 2 & 0.3285 & 5.5739 & 4.0315 \\
\hline Segment 3 & 0.9943 & 5.1775 & 2.9602 \\
\hline Segment 4 & 0.2348 & 3.2549 & 2.9614 \\
\hline Segment 5 & 5.4488 & 4.6115 & 3.7545 \\
\hline
\end{tabular}

Table 3. Value of Entropy for segments of Polygons, Lena and Space

As it can be seen in Table 3, the Entropy of segments of the "Lena" image has the highest degree of Entropy which may sound unusual, because the image "Space" 
as a whole has a higher degree of Entropy, a rate and a level of detail. However, this corroborates that in images in which a larger number of segments are detected, Entropy as a measure of the potential of the image and an "ability" of segments, decreases. There is no a universal rule that a large number of segments should be detected in the case of images with a high level of detail. However, the image may possess a high degree of change, which is almost always below the prescribed threshold. Therefore, if it is necessary, the proposed algorithm may redefine the threshold detection and directly adjust desired needs. For example, in cases of extremely intense lighting the threshold detection can be regulated to an upper limit of bit entries, so that parts of the image with a high degree of light do not affect the other parts of the image segmentation.

The segmentation method, which is proposed in this research paper, provides a new technique which is different from the following:

1. Clustering methods - the proposed technique does not select pixels at random but examines the environment based on them in order to define a segment,

2. Thresholding - the proposed method does not only analyse greyscale but it also uses $2 \mathrm{D}$ gradient,

3. Compression-based methods - the proposed method is not based on the segment minimisation and does not use the compression,

4. Partial differential equation-based methods - the proposed method is a much simpler segmentation method and it is not used for recognition purposes,

5. Region-growing methods - unlike in this method in which the segments are graded on the basis of similarity between surrounding pixels, the proposed method defines segments within the defined edges,

6. Dual clustering method - this method is based on the bit plane, so it is limited in comparison to the edge detection of each bit plane,

7. Histogram-based methods - though very effective in certain situations because the segments are detected on the basis of belonging to a particular part of the spectrum, this method is not effective if there are several segments that are distant from each other but have similar or the same colour shades.

\section{CONCLUSION}

According to all of the above facts of the study, this kind of segmentation uses a mixture of two edge detection methods. In the first section, the parallel detection was used for the comparison of the original image and the image with the Sobel edge detection. On the other hand, in the second part, the sequential detection based on the pixel as a starting mark for detection is used. The last pixel of the first segment is not the starting pixel of the second segment, as this would violate one of the basic segmentation rules - segments cannot be overlapped. The first next pixel with the value less than 125 will be the starting point for the next segment. In this case a possible error of segmentation can be observed, because of the separate segments which do not contain the edge defined by the Sobel algorithm. If this is taken as an error of segmentation, then the images with higher LoD can make a bigger error. This problem can be relatively overcome by reducing the level of threshold. It is certain that, this issue can be the basis for some further considerations. Furthermore, for different purposes, further considerations may be directed to the use of other algorithms for detecting edges in different kind of sequential detection threshold. It should be particularly emphasized that the precision of the proposed algorithm is at the level of pixel, which is considered as a huge advantage.

After exploring the static image as a fundamental element of multimedia, the future research will move in the direction of the segmentation of video recordings. Debugging, which may occur in image Figure $9 g$ and Figure $9 \mathrm{~h}$. Furthermore, the connection with algorithms for the detection and identification, and/or color Doppler aiming to monitor specific forms [21].

\section{DISCLOSURES}

No conflicts of interest, financial or otherwise, are declared by the authors.

\section{ACKNOWLEDGMENT}

This work was done within the EU project - Implementation of the study program - Digital Broadcasting and Broadband Technologies (DBBT Master studies), Erasmus+ CBHE Project No. 561688-EPP1-2015-1-XK-EPPKA2-CBHE-JP. 


\section{REFERENCES}

[1] S. Lu, S. Wang, Y. Zhang, "A note on the markerbased watershed method for X-ray image segmentation", Computer Methods and Programs in Biomedicine, 141, pp. 1-2, 2017.

[2] S.-H. Wang, J. Sun, P. Phillips, G. Zhao, Y.-D. Zhang, "Polarimetric synthetic aperture radar image segmentation by convolutional neural network using graphical processing units", Journal of RealTime Image Processing, 1-12 (2017).

[3] P. Kałamała, A. Sadowska, W. Ordziniak, A. Chuderski, "Gestalt Effects in Visual Working Memory", National Center for Biotechnology Information, U.S. National Library of Medicine, Experimental Psychology, vol. 64, no.1, pp. 5-13, 2017.

[4] M. Wertheimer, Gestalt theory. In A source book of Gestalt psychology, Ch. 1, Ed. W. D. Ellis. New York: The Humanities Press, 1938.

[5] E. Cuevas, D. Zaldívar, G. Pajares, M. Perez-Cisneros, R. Rojas, "Computational Intelligence in Image Processing 2016", Mathematical Problems in Engineering 2016, Article ID 5680246, pp. 1-3, 2016.

[6] C. Park, J. Z. Huang, J. X. Ji, Y. Ding, "Segmentation, inference and classification of partially overlapping nanoparticles", IEEE transactions on pattern analysis and machine intelligence, vol. 35 , no.3, pp. 1-21 (2013.

[7] S. Patil, A. Junnarkar, "Overview of Colour Image Segmentation Techniques", International Journal of Advanced Research in Computer Science and Software Engineering, vol. 3, pp. 418-423, 2013.

[8] D. M. Muñoz, C. Franciscangelis, W. Margulis, F. Fruett, I. Söderquist, "Low latency disturbance detection using distributed optical fiber sensors", IEEE 14th International Conference on Networking, Sensing and Control (ICNSC), vol. 14, no. 1, pp. 1-6, 2017.

[9] B. Jaksic, R. Ivkovic, B. Gara, M. Petrovic, P. Spalevic, "Analysis of different influence of compression algorithm on the image filtered Laplcian, Prewitt and Sobel operator", International Journal of Darshan Institute on Engineering Research and Emerging Technology, vol. 2, no.1, pp. 59-67, 2013.

[10] P. P. Acharjya, R. Das, D. Ghoshal, "A study on image edge detection using the gradients", International Journal of Scientific and Research Publications, vol. 2, no.12, pp. 1-5, 2012.
[11] R. C. Gonzalez, R. E. Woods, S. L. Eddins, Digital Image Processing Using MATLAB, Prentice Hall, Upper Saddle River, 2004.

[12] J. Lee, R. M. Haralick, L. G. Shapiro, "Morophologic edge detection", IEEE Journal of Robotics and $\mathrm{Au}$ tomation, vol. 3, no. 2, pp. 142-156, 2003.

[13] W. P. Y. Shanlin, "Gray Image's Egde Detection Based on Gauss-Laplace", Computer Engineering and Applications, Institute of Computer Network System, vol. 26, no. 1, pp. 2-23, 2003.

[14] R. Ivkovic, M. Petrovic, B. Gara, M. Milosevic, "Dental Image Analysis with Different Edge Detection Operators", Journal of Communications Technology and Electronics, Pleiades Publishing, Inc., vol. 59, pp. 1289-1297, 2014.

[15] B. Jaksic, R. Ivkovic, B. Gara, M. Petrovic, P. Spalevic, "Analysis of different influence of compression algorithm on the image filtered Laplcian, Prewitt and Sobel operator", International Journal of Darshan Institute on Engineering Research and Emerging Technology, vol. 2, no. 1, pp. 59-67, 2013.

[16] R. Ivkovic, I. Miloševic, B. Gara, S. Minic, V. Dakovic, "Algoritam za detekciju ivica slike sa postfiltrom”, XIII medunarodni naucno-strucni simpozijum Infoteh 2014, Jahorina, Bosna i Hercegovina, vol. 13, no. 1, pp. 652-656, 2014.

[17] S. Vijayarani, M. Vinupriya, "Performance analysis of Canny and Sobel edge detection algorithms in Image Mining," International Journal of Innovative Research in Computer and Communication Engineering, vol. 1, no. 8, pp. 1760-1767, 2013.

[18] S. Ilic, M. Petrovic, B. Jaksic, P. Spalevic, Lj. Lazic, M. Milosevic, "Experimental analysis of picture quality after compression by different methods", Przegląd elektrotechniczny, vol. 89, vol. 11, pp. 190194, 2013.

[19] S.R. Cloude, E. Pottier, "An entropy based classification scheme for land applications of polarimetric SAR", IEEE Trans. on Geoscience and Remote Sensing, vol. 75, no. 1, pp. 68-78, 1997.

[20] 20.J. Beirlant, EJ. Dudewicz, L. Gyorfi, E. van der Meulen, "Nonparametric entropy estimation: an overview", International Journal of Mathematics \& Statisticsis, vol. 6, no. 1, pp. 17-39, 1997.

[21] 21.L. Kopanja, S. Kralj, D. Zunic, B. Loncar, M. Tadic, "Core-shell superparamagnetic iron oxide nanoparticle (SPION) clusters: TEM micrograph analysis, particle design and shape analysis", Ceramics International, vol. 42, no. 9, pp. 1097610984, 2016. 